\title{
Primeiro encontro de Lutzomyia longipalpis (Lutz \& Neiva, 1912) na área urbana de Uberlândia, MG, concomitante com o relato de primeiro caso autóctone de leishmaniose visceral humana
}

\author{
First finding of Lutzomyia longipalpis (Lutz \& Neiva, 1912) in \\ the urban area of Uberlândia, MG, concomitant with the first \\ reported autochthonous case of human visceral leishmaniasis
}

\author{
Márcia Beatriz Cardoso de Paula ${ }^{1}$, Elisângela de Azevedo Silva Rodrigues ${ }^{1}$, \\ Amaral Alves de Souza ${ }^{1}$, Alessandro Ambrosio dos Reis ${ }^{1}$, Flávio Peixoto de Paula ${ }^{1}$, \\ Adalberto de Albuquerque Pajuaba Neto ${ }^{1}$ e Jean Ezequiel Limongi ${ }^{1}$
}

\begin{abstract}
RESUMO
Relata-se a primeira ocorrência do vetor da leishmaniose visceral, Lutzomyia longipalpis, na área urbana de Uberlândia, Estado de Minas Gerais e o primeiro caso de leishmaniose visceral humana autóctone no município, notificado ao Centro de Controle de Zoonoses, por meio da Vigilância Epidemiológica da Secretaria Municipal de Saúde. Discute-se a importância deste encontro na transmissão da doença nessa área.
\end{abstract}

Palavras-chaves: Leishmaniose visceral. Transmissão. Ecologia de vetores. Lutzomyia longipalpis.

\begin{abstract}
The first occurrence of the vector for visceral leishmaniasis, Lutzomyia longipalpis, in the urban area of Uberlândia, State of Minas Gerais, and the first autochthonous case of human visceral leishmaniasis recorded in the same locality are reported. These were notified to the Zoonosis Control Center, through the Epidemiological Surveillance sector of the Municipal Health Department. The importance of these findings regarding transmission of the disease in this area is discussed.
\end{abstract}

Key-words: Visceral leishmaniasis. Transmission. Ecology of vectors. Lutzomyia longipalpis.

Lutzomyia longipalpis (Lutz \& Neiva, 1912) é o principal transmissor da Leishmania chagasi, agente etiológico da leishmaniose visceral. Recentemente, verificou-se que este flebotomíneo está bem adaptado, principalmente na região sudeste, às periferias de grandes centros e aos ambientes urbanos. Também, tem sido encontrado no intradomicílio e, no peridomicílio, em galinheiros, chiqueiros e canis, entre outros ambientes ${ }^{1}$.

De acordo com informações obtidas no Sistema de Informações de Agravos de Notificação (SINAN), do Ministério da Saúde do Brasil, 398 casos de LV humana foram notificados em Minas Gerais no ano de 2007 (dados parciais). Em Uberlândia, um caso de LV humana foi diagnosticado em 2004, não sendo, porém, confirmado como sendo autóctone.
A investigação da fauna flebotomínica no município de Uberlândia tem sido sistematicamente realizada pelo Centro de Controle de Zoonoses (CCZ), da Secretaria Municipal de Saúde, desde 1998. Várias espécies de flebotomíneos incriminadas como transmissoras de leishmaniose tegumentar americana (LTA) foram capturadas na zona rural e urbana da cidade. Em algumas localidades rurais, identificou-se a presença de 131 espécimes de Lutzomyia longipalpis, no período de 2003 a 2007, classificando o município, segundo critérios para definição de áreas de transmissão de LV do Ministério da Saúde, como área silenciosa e vulnerável para LV, porém, receptiva em sua zona rural ${ }^{123}$.

O Município de Uberlândia está localizado na porção sudoeste do Estado de Minas Gerais, região do Triângulo Mineiro, entre as coordenadas geográficas de 18056'38" de latitude sul e 48018'39"

1. Centro de Controle de Zoonoses, Secretaria Municipal de Saúde de Uberlândia, Uberlândia, MG.

Endereço para correspondência: Dr ${ }^{\text {a }}$ Márcia Beatriz Cardoso de Paula. Av. Alexandrino Alves Vieira 1423, Bairro Liberdade, 38401-240 Uberlândia, MG.

Telefax: $55343213-1470$

e-mail: mbecpaula@yahoo.com.br

Recebido para publicação em: 27/02/2008

Aceito em: 02/06/2008 
de longitude oeste de Greenwich, a uma altitude média de $863 \mathrm{~m}$ [fornecida pelo Instituto Brasileiro de Geografia e Estatística (IBGE)]. Ocupa uma área total de $4.115,09 \mathrm{~km}^{2}$, sendo que $219,00 \mathrm{~km}^{2}$ são ocupados pela zona urbana e $3.896,09 \mathrm{~km}^{2}$, pela zona rural. 0 clima é tropical chuvoso, com inverno seco, sendo que a precipitação média anual é de 1500-1600mm, com forte concentração de chuvas nos meses de dezembro a fevereiro. A temperatura média mensal varia de $20,9^{\circ} \mathrm{Ca} 23,1^{\circ} \mathrm{C}$ e o período mais quente do ano se estende de outubro a março. A vegetação é típica de cerrado e a hidrografia bastante rica. A população de Uberlândia foi estimada, em 2007, em 608.369 habitantes. Uberlândia constitui-se num importante entroncamento rodo-ferroviário, que facilita a comunicação com os principais centros urbanos das regiões Sudeste e Centro-Oeste.

Em janeiro de 2008, foi notificado ao CCZ, por meio da Vigilância Epidemiológica da Secretaria Municipal de Saúde, um caso de IV humana em uma criança de seis meses e 22 dias de idade, natural e procedente de Uberlândia, que, ao exame físico apresentava sintomas de febre, anorexia, hipoatividade, palidez cutânea, edema difuso, linfadenomegalia, esplenomegalia, hepatomegalia e pancitopenia. № mielograma, corado pela técnica de Papanicolaou, foram visualizadas formas amastigotas de Leishmania sp (Figura 1). Em adição, foi realizada a sorologia utilizando a técnica de imunofluorescência indireta (RIFI) que apresentou resultado não reativo. A criança recebeu tratamento com anfotericina B lipossomal, apresentando melhora clínica e tendo alta hospitalar em fevereiro de 2008.

Investigações epidemiológicas confirmaram que o paciente não apresentava histórico de viagem a áreas onde a transmissão

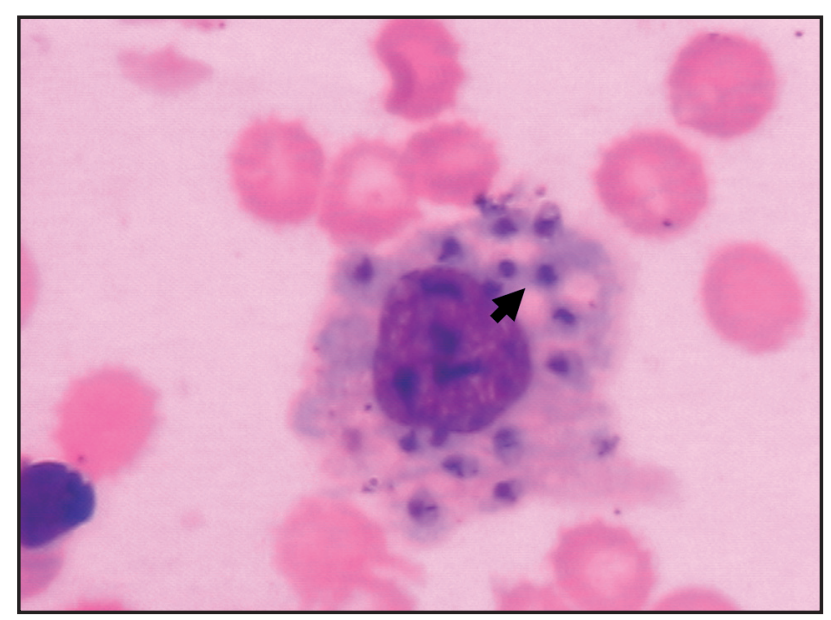

Figura 1 - Formas amastigotas de Leishmania sp no interior de macrófago da medula óssea. 100X.

de LV é endêmica. Diante da suspeita de autoctonia de LV foi iniciada, pelo Laboratório de Entomologia do CCZ, uma investigação entomológica com o objetivo de verificar a presença de Lutzomyia longipalpis. As capturas foram realizadas durante três dias consecutivos na residência do paciente e em outras 12 residências vizinhas. A metodologia de captura utilizada foi com armadilhas luminosas do tipo CDC (Center for Disease Control), instaladas no peri e intradomicílio, e capturadores à base de sucção do tipo Castro. 0 período de exposição das armadilhas CDC foi das 18:00 às 7:00 horas do dia seguinte. Os capturadores do tipo Castro foram utilizados uma hora após o crepúsculo, com um período mínimo de pesquisa de 30 minutos/domicílio, sendo 15 minutos para a coleta no intradomicílio e 15 minutos para o peridomicílio ${ }^{1}$.

Os flebotomíneos capturados foram identificados segundo a chave de identificação de flebotomíneos de Young \& Duncan ${ }^{4}$, após a montagem. Na residência do paciente, foram capturados seis espécimes de Lutzomyia longipalpis, cinco machos e uma fêmea, no peridomicílio, próximo ao local de abrigo de cães. Em outras cinco residências vizinhas, foram capturados cinco espécimes de Lutzomyia longipalpis, sendo quatro machos e uma fêmea. A área do caso está localizada no Bairro Ipanema, próximo ao aeroporto do município, sendo considerada como região periurbana da cidade.

Este é o primeiro relato de leishmaniose visceral humana autóctone no município de Uberlândia e o primeiro encontro de Lutzomyia longipalpis na zona urbana. No bairro onde ocorreu a doença humana reside um grande número de pessoas que vieram de áreas sabidamente endêmicas para LV. Desta forma, é possível que cães infectados tenham vindo para o município acompanhando seus donos e, conseqüentemente, infectado os flebotomíneos que estão começando a se urbanizar, vindos da área da mata adjacente ao bairro. Um inquérito canino censitário está sendo realizado naquele bairro, com coleta de sangue por meio de punção da veia marginal auricular, para diagnóstico da LV canina pelo método de imunofluorescência indireta. Outras medidas de controle e prevenção estão sendo reforçadas para evitar que a doença se estabeleça em todo o município, como vem acontecendo em outras regiões do Brasil.

\section{AGRADECIMENTOS}

Agradecemos a Daniel Alves Leal, Iram Martins Costa, Jovenil Gomes da Silva, Mauro Santos Lima e Wilson Gomes Moreira, do Laboratório de Entomologia do Centro de Controle de Zoonoses de Uberlândia, pela contribuição nas atividades de campo e de laboratório, imprescindíveis para o desenvolvimento deste trabalho. Agradecemos ao Dr. Eduardo Henrique Roscoe pela notificação do caso ao Serviço de Epidemiologia Municipal. Agradecemos Leonardo Bruno Figueiredo pela confecção da foto.

\section{REFERÊNCIAS}

1. Fundação Nacional de Saúde. Manual de Vigilância e Controle da Leishmaniose Visceral. Ministério da Saúde. Brasília, 2006.

2. Paula MBC, Reis AA, Silva EA, Limongi JE, Neto AAP. Levantamento da fauna flebotomínica do município de Uberlândia-MG, Brasil, no período de 2003 a 2004, utilizando duas diferentes armadilhas luminosas e inquérito soroepidemiológico canino. In: Resumos do XXXII Congresso Brasileiro de Medicina Veterinária, Uberlândia p. 22, 2005.

3. Paula MBC, Reis AA, Silva EA, Silva AA, Lopes IS, Limongi JE, Neto AAP. Estudo Comparativo entre duas diferentes armadilhas luminosas utilizadas em investigações entomológicas no município de Uberlândia-MG, realizadas no período de 2003 a 2004, e inquérito soroepidemiológico canino para Leishmanioses. In: Resumos do XXI Reunião Anual de Pesquisa Aplicada em Doença de Chagas e Leishmanioses, Uberaba p. 6, 2005.

4. Young DG, Duncan MA. Guide to the identification and geographic distribution of Lutzomyia sand flies in Mexico, the west Indies, Central and South American (Diptera: Psychodidae). Memories American Entomology Institute 54:1-881, 1994. 